\title{
Multimodal Brain Images Fusion using Cultural Algorithm Optimized Multispectral Features
}

\author{
Deepti Nathawat, Manju Mandot, Neelam Sharma
}

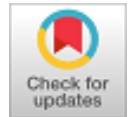

\begin{abstract}
Medical images can be acquired through different techniques (modalities), which have their own application areas; some of them provide information on the functional activity, while others contain only anatomic information. Usually, in the first case, images have low spatial resolution while in the second case have a higher resolution. However, the analysis of medical images often requires the evaluation of more than one modality; in order provide the specialist with more information for decision making as well as for the analysis and the treatment of diseases. Image fusion aims to combine information from the same sensor or different sensors, so that the image fused retain the information content of each individual image. In remote perception, when multispectral images are analyzed, it is very important to preserve the content of spectral information of each of the bands. The challenge is to obtain good quality images that allow us to extract as much amount of information possible, for which it is sometimes necessary to enhance or modify the image to improve its appearance or combine images or portions thereof to combine the information. An ideal fusion of multispectral images and the band panchromatic will result in a new series of bands with greater spatial resolution and equal spectral content. This paper proposes a PCA, DWT and cultural optimized entropy based DWT fusion with the evaluation parameters; arithmetic mean $(S M)$, Maximum value $\left(V_{\max }\right)$ and Minimum value $\left(V_{\min }\right)$.
\end{abstract} etc.

Keywords - PCA, DWT, CULTURAL, SM, $V_{\max }$ and $V_{\min }$

\section{INTRODUCTION}

In a clinical study or diagnosis, many sources information is made available to the clinician: different imaging modalities, and different tools to better study the structure or pathology. The clinician should perform the synthesis of these different information in order to achieve a diagnosis and / or a precise and reliable treatment. However, this synthesis can be long, tedious and remains very dependent on the operator who is charge. In this context, image fusion appears as a new tool to help diagnosis; making the task easier for the doctor by providing a simpler fusion tool. So, this heading will be articulated in three parts, at first we will present the imagery medical with its different modalities, then we will approach the image fusion and its different approaches and we will end up with state of the art work on fusion.

Revised Manuscript Received on December 30, 2019.

* Correspondence Author

Ms. Deepti Nathawat*, Department of Computer Science, Banasthali Vidyapith, Vanasthali, Niwai, Rajasthan, India, Email: er.deeptichauhan@gmail.com

Prof. Manju Mandot, Department of Computer Science, Rajasthan Vidyapith, Udaipur, Rajasthan, India, Email: manju.mandot@gmail.com

Dr. Neelam Sharma, Department of Computer Science, Banasthal Vidyapith, Vanasthali, Niwai, Rajasthan, India, Email: sharmaneelam27@gmail.com

(C) The Authors. Published by Blue Eyes Intelligence Engineering and Sciences Publication (BEIESP). This is an open access article under the CC BY-NC-ND license (http://creativecommons.org/licenses/by-nc-nd/4.0/)
Several fusion techniques have been proposed, such as intensity-maturation, principal component analysis, and fusion methods with the Wavelet transform (WT) [1]. The fusion process consists of calculating the wavelet transform of each image, apply the fusion rules based on measures of correspondence and outgoing, combine the coefficients of the WT to generate a just set of coefficients and finally recover the composite image to through the inverse WT transform.

The efficiency of the proposed WT approach was compared with other techniques of fusion such as principal component analysis and WT from methods statistics. The information content of the images resulting fused was compared with the information content before the fusion to assess its integrity.

On the basis of various studies that reveal important advances in diagnostic diagnosis medical image caused by the presence of medical images that resulted of the process of fusing a pair of images used at the beginning, the convenience of undertaking a comprehensive study within the field of fusion of images in contexts of medical image.

This project is aimed at priority the implementation and subsequent evaluation of different fusion algorithms of medical images. To achieve this $n$, sets of sets of image pairs have been used to proceed to fuse by implementing several different algorithms, in order to reach both objective conclusions, based on some conclusive quantitative data, such as the signal to noise ratio or the correlation coefficient, among others, as subjective, through the opinion of an optional specialist in Radiology, who has expressed his opinion on what images natives already fused are more friendly for a better diagnosis of the possible patient ailment The algorithms that have been implemented are those known as multi resolution algorithms, which belong to the category of pixel level, and whose operation is based on the pyramidal decomposition of the starting images, obtaining replicas of them at different resolutions. The complete fusion process that has been developed in this work consists of take a couple of original images from different radiological technologies and related to the same anatomical cut in a given patient, and once registered both pass each of them separately to a transformed domain, specifically to the wavelet domain for the case at hand, by means of the wavelet transform two-dimensional discrete (DWT), and subsequently apply one of the fusion rules that have been developed to these transformed images. Finally you get the final image result of the fusion using the inverse discrete wavelet transform (IDWT) [2]. 


\section{LITERATURE REVIEW}

The memory consists of a state of the art which it is done a brief tour of the beginnings of Radiology and the main comments are made Medical imaging technologies. The process is also introduced in an introductory way of registration of images, since to be able to fuse a pair of images. Fusion has become an important aspect of information processing in several fields [3]. Several senses are given to the fusion, we take here the definition proposed [4]. The fusion information is to combine information from multiple sources to help decision. Fusion is utilized for diagnosis and treatment in image processing field [5].

The fusion of data covers a broad domain of applications and methods, so it is very difficult to provide a precise definition and sometimes it is often confused with other terms that refer to the same concept but in a more general sense, such is the case of the combination and mixing data [6], establishing that "data fusion is a multilevel and multi-faceted process that deals with automatic detection, association, correlation, estimation and combination of data from multiple sources" [7] [8] [9]. The fusion of medical images is then defined as the combination of images that come from various modalities in a single image with a greater amount of information, with the purpose of presenting all the information (anatomical and functional) in a joint and integrated way to simplify its interpretation and given that the images used in this work are two-dimensional.

\section{MEDICAL IMAGE FUSION}

In general, the purpose of the fusion process is to integrate different data in the end goal to collect more information than can be attained from each individual data sensor. Also, data fusion provides a robust operational task, for example, increased reliability, reduced ambiguity, improved credibility and classification.

In the literature [3] it has been found, image fusion for the following purposes:

- Image Enhancement: Spatial resolution can be increased utilizing the image fusion.

- Improve Geometric Corrections (Registration Accuracy): The fusion offers the advantage of an integral rectification approach that improves the accuracy in the register since the location of the control points for the registration is simplified if they can be located in analogous outlooks.

- Classification is improved by complimenting the data sets. If you have data from different sources you can make better class discrimination.

- Detection of changes in multi temporal data.

- Replacement of information omitted in an image having other sensor signals.

- Replacement of defective data.

The primary objective of medical image fusion is to increase the quality of care in the face of diagnostic and planning processes:

- Facilitating and advancing the diagnosis through the use of functional / anatomical image fusion techniques

- Increasing the planning accuracy in radiotherapy,
- Allowing a quality control of radiotherapy treatment based on objective measurements [10]. Generally, the image fusion system is categorized into following approaches: image acquisition, registration, pre-processing, fusion, post-processing and deployment as presented in Figure 1. The first approach is registration of images with respect to reference image.

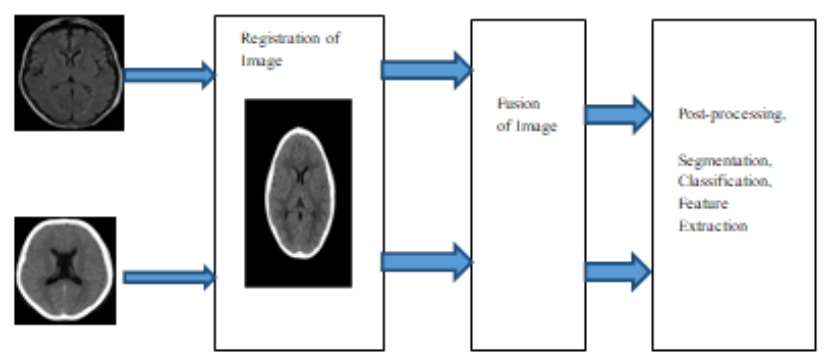

Figure 1: Generalized block diagram for image fusion

Registration of image will ensure the proper alignment of image to be fused. The upcoming paragraph provides the generalized procedure for image fusion.

Initially, the information collected from various input resources arrives in the registration phase which combines the equivalent pixels of the similar physical points. This realizes the pixel by pixel comparison of the input images. Consequently, the data may enter a preprocessing stage (if necessary) for noise reduction. Then the preprocessed images enter the fusion method. One can use any particular fusion method here and result of that method provides a certain variety of image; composite, thematic map or 3Dgraph. If the fusion is applied on the pixel level then there must be a post-processing phase applied on the fused image for example restoration, classification or segmentation. Finally the performance is evaluated by using certain parameters.

Image subsystems are composed of multiple (usually two) sensors of pictures; in medical images for example, these sensors refer to some modality [11]. Given the complexity of image registration, the objective of this work focuses on the fusion subsystem; consequently, it is assumed that the images used for the evaluation of the proposed technique are already registered.

Pre-processing before or after fusion is a generally desirable step since that most fusion algorithms highlight artifacts from the original data, thus obtaining undesirable results in the last stage. Instead, post-processing it will depend on the type of deployment and the purpose of the fusion system. Finally, the deployment subsystem shows the fused image to the end user. The quality of this output image will depend largely on the fusion process used, the optional post-processing and deployment type.

Data fusion takes place at three levels: pixel, characteristics, and decision [12]. In pixel-level fusion, images from different sources are combined pixel by pixel, which is why this type of fusion represents the minimum level of processing to fuse physically measured parameters [13].

Published By:

Retrieval Number: A2080109119/2019@BEIESP

DOI: 10.35940/ijeat.A2080.129219

Journal Website: www.ijeat.org

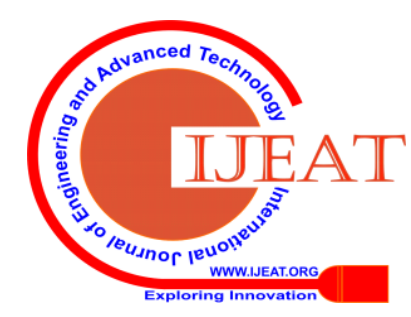


In general, the algorithms used at this level are easier to implement and more efficient from the computational point of view, so almost all the fusion algorithms belong to this level [14], and it is precisely in this category that find the three-dimensional fusion algorithm of this paper.

Fusion at the feature level requires the extraction of relevant features in the input images. These characteristics can be intensities of pixels, borders or textures depending on the nature of the images. Similar characteristics are compared to each other and then fused for future evaluations using statistical approaches or neural networks. Finally, fusion at the decision level allows the combination of images at the highest level of abstraction. The input images are processed independently and their results are combined using decision rules based on fuzzy logic, statistical methods, etc. The information obtained from combining the images, applying decision rules, allows reinforcing a common interpretation, resolving differences and providing a better understanding of the observed object. Depending on which method is used, the fusion is classified as hard fusion or soft fusion [15].

\section{IMAGE FUSION TECHNIQUES}

There are a lot of algorithms proposed to solve the problem of image fusion, and especially to make the fusion at pixel level and often with specific adaptations for particular problems. Because of this it is possible to find in the literature various categorizations of fusion algorithms.

The main techniques of image fusion will be presented in the following order:

\section{A. Arithmetic Combinations}

\section{Weighted combination (Sum And Multiplication)}

For contrast enhancement, the addition and multiplication of images give good results [15]. An example of the multiplication process is expressed by the equations:

$$
\begin{aligned}
& \mathrm{DN}_{\mathrm{f}}=\mathrm{A}\left(\mathrm{w}_{1} \mathrm{DN}_{\mathrm{a}}+\mathrm{w}_{2} \mathrm{DN}_{\mathrm{b}}\right)+\mathrm{B} \\
& \mathrm{DN}_{\mathrm{f}}=\mathrm{ADN}_{\mathrm{a}} \mathrm{DN}_{\mathrm{b}}+\mathrm{B}
\end{aligned}
$$

$A$ and $B$ are scale factors and $\mathrm{w}_{1}$ and $\mathrm{w}_{2}$ are weight parameters. $\mathrm{DN}_{\mathrm{f}}, \mathrm{DN}_{\mathrm{a}}$ and $\mathrm{DN}_{\mathrm{b}}$ refer to the values of the fused final image and the input images a and $b$ respectively.

\section{Average}

When two images are combined, an intuitive approach is to take the average of the pixels of the source images to obtain the corresponding pixel value in the composite image. However, when this method is taken directly, the contrast of the objects present in the images is reduced. To avoid the problem of averaging fuse, the fused image can be obtained by selecting pixels from its source images.

\section{Simple Average}

This approach is the simplest fusion techniques and consists of finding the average value of the intensity of the input images. For example, for the case of two images, the value of the pixel $P(m, n)$ of each of the images is taken, added, and then divided by 2 . The result of the said division allocate to the corresponding pixel of the image of merged output. Equation (3) shows the general operation of this technique, where $\mathrm{S}$ is the resulting image, $I_{k}$ is the input images and $k$ is the number of input images.

$$
F(m, n)=\frac{1}{k} \sum_{k-1}^{k} I_{k}(m, n)
$$

\section{Maximum Value}

In this technique, the resulting image acquired by selecting the maximum intensity value correlating to both images, because the regions of the images that are focused tend to have higher intensity pixels.

\section{Minimum Value}

$$
F(i, j)=\operatorname{MAX}\left\{I_{1}(i, j) I_{2}(i, j)\right\}
$$

In this technique, the resulting image is acquired by selecting the minimum intensity value correlating to both images to be merged.

$$
F(i, j)=M I N\left\{I_{1}(i, j) I_{2}(i, j)\right\}
$$

\section{B. Principal Component Analysis (PCA)}

The principal component method (PCA: principal component analysis) is a statistical method that transforms a set of correlated variable data into a new group of uncorrelated data, obtained by linear combination of the originals [5]. The calculation of the main components (PC) includes the use of the covariance matrix or the correlation matrix for the calculation of eigenvalues or eigenvectors and the calculation of the main components (Figure 2).

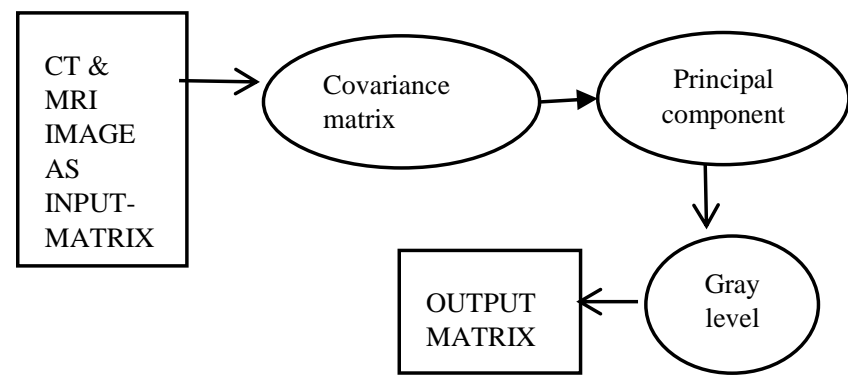

\section{Figure 2: Diagram of the transformation of main components}

In general terms, the first two or three main components contain between $95 \%$ to more than $99 \%$ of the information contained in the scene. Therefore, PCA is a way to identify patterns in the data and express them differently to highlight their similarities and differences, while reducing the image dimension without significant loss of information.

For the fusion process, multispectral images are first transformed into a group of new bands that are not correlated, the first component PC1 contains information that is common in all multispectral bands, while spectral information is detailed in the other components. Subsequently, these components are combined to obtain a new set of main components, to which the PCA Reverse Transform is applied and thereby the fused image is finally obtained.

Image fusion using PCA has two approaches [16]:

1. Substitution: Replacement of the first main component with another image (Principal Component Substitution, PCS).

2. Combination of main components of all images of the different sensors.

The first approach follows the idea of increasing the spatial resolution of the multi-channels of the image by introducing an image with high resolution. The channel that replaces PC1 is normalized to the variance of this first component and averaged. 
A high image resolution value replaces PC1, which is common in all bands while the spectral information is unique to each band. The first PC1 has a maximum variance which can maximize the effect of high data resolution on image fusion.

The second procedure integrates the disparate nature of the multi-sensor input data into an image. The bands of the image of different sensors are combined in a file and a PCA is calculated from all bands [12].

PCA fusion methods are sensitive to the choice of the analysis area because the covariance and correlation matrix are specific to the geographic extent selected. On the other hand, the correlation coefficient reflects a close relationship in homogeneous samples, however, changes in the values of the bands due to the marked differences in the types of coverage also influence the correlations and particularly in the variances.

The basic idea of multiresolution fusion is to effect a multiscale transformation (MST: Multi-scale Transform) on each original image, then construct a multiresolution representation. The fused image is obtained by taking the inverse multiscale transform (IMST: Inverse Multi-scale Transform) [17].

The most commonly used multiresolution methods are the pyramidal transform and the discrete wavelet transform (DWT). The wavelet transform, which is the method proposed in this paper, can also be used as a multi-resolution technique, and there are many more like curvelets, contourlets, wedgelets, ridgelets. In addition to the technique of multiresolution analysis [18], another important aspect is the way in which the coefficients, so some general alternatives for the construction of the fusion rule in this type of techniques.

\section{Wavelet Decomposition}

The analysis based on the wavelet theory, allows the two-dimensional decomposition of the image into different frequency components, and different resolutions.

The wavelet analysis described above is known as the continuous wavelet transform (CWT: Continuous Wavelet Transform) and is formally written as:

$$
W_{x}^{\psi}(\tau, s)=<x, \psi_{s, \tau}>=\frac{1}{\sqrt{|S|}} \int x(t) \psi^{*}\left(\frac{t-\tau}{s}\right) d t
$$

Where: $S$ and $t$ are parameters of scale and translation. $x(t)$ is the signal to which the transform is applied. The $\psi$ function and $(t)$, is the transformation function and is called the mother wavelet, which It is a finite length oscillating waveform that is used as a prototype to generate the other window functions (scaled and translated copies) called "daughter wavelets". For each scale s and location t, the wavelet coefficients represent the information contained in $x(t)$, so the original signal can be exactly reconstructed from the coefficients of wavelet by:

$$
x(t)=\frac{1}{c_{\psi}} \int_{0}^{\infty} \int_{+\infty}^{\infty} W_{x}^{\psi}(s, \tau) \psi_{s, \tau} d \tau \frac{d s}{s^{2}}
$$

Where $C_{\psi}$ is the normalization factor of the mother wavelet.

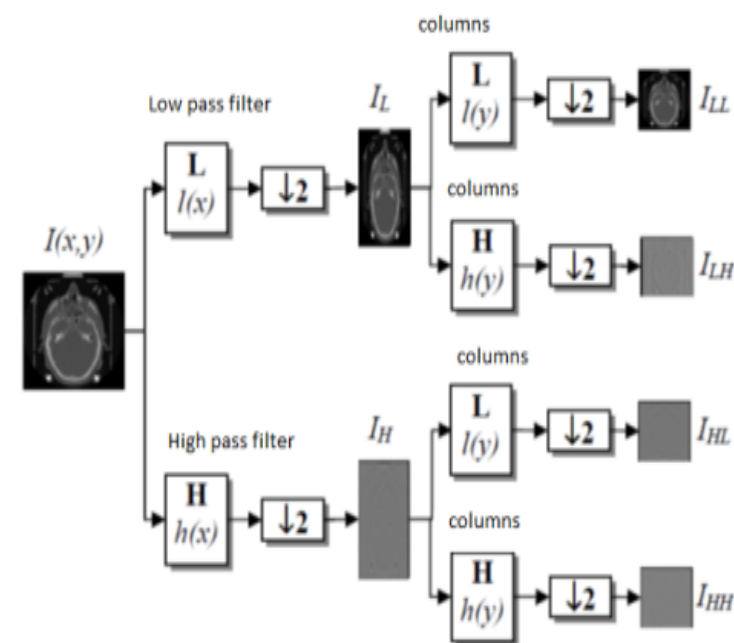

Figure 3: A stage of the decomposition of an image using DWT [17]

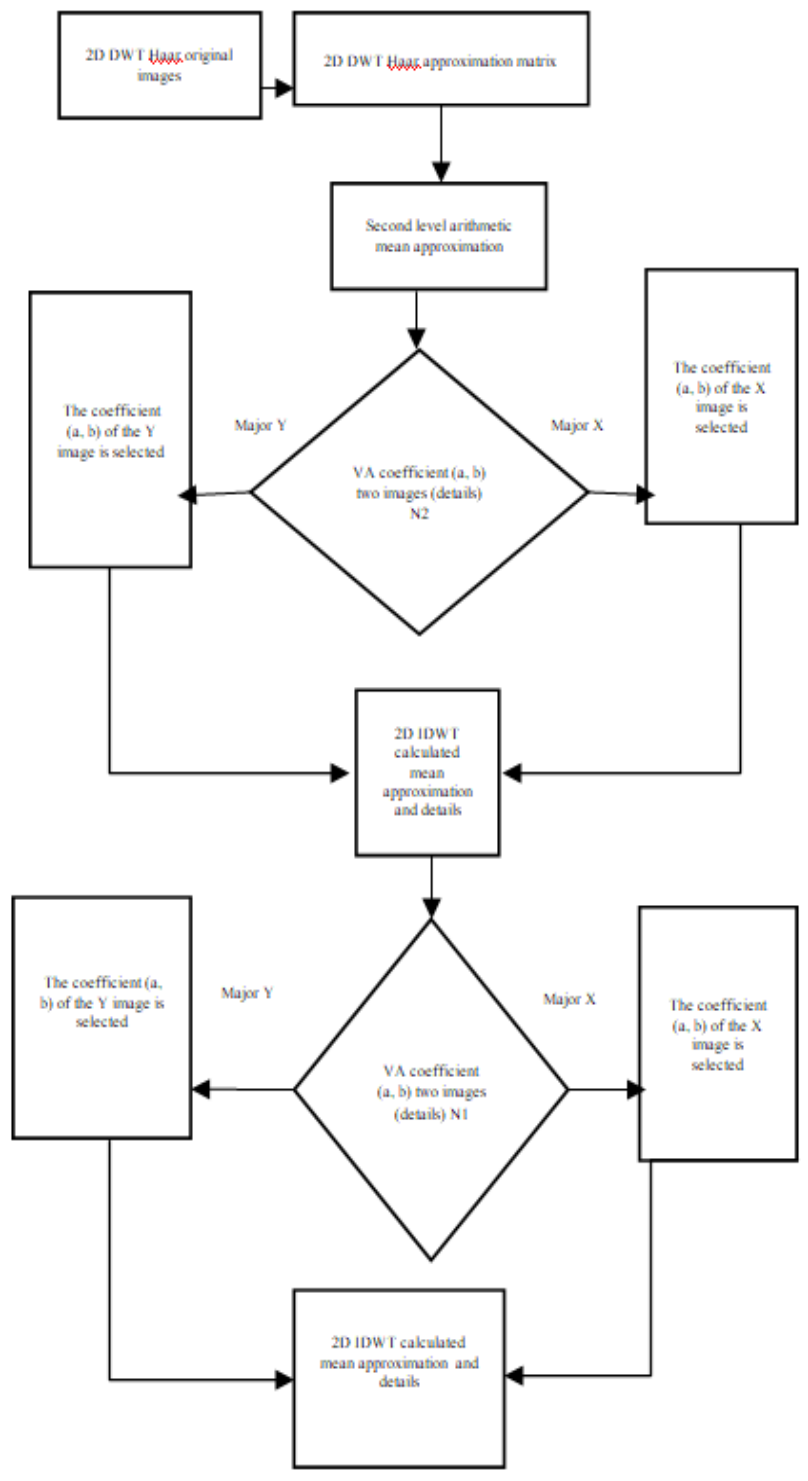

Figure 4: Breakdown of the block level of second level activity

Published By:

Retrieval Number: A2080109119/2019@BEIESP

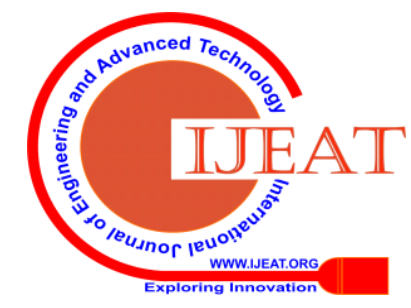


There are different families of wavelets functions, which differ in how the basic functions and how smooth they are located in space. Within a family of wavelets are the subclasses, distinguished by the number of coefficients and by the level of iteration.

Due to the above, the first wavelet-based fusion schemes [19] reported results superior to standard pyramidal methods, both qualitative and quantitatively. However, DWT is not invariant to translation due to subsampling used in its construction and this causes ring artifacts and movement in the images.

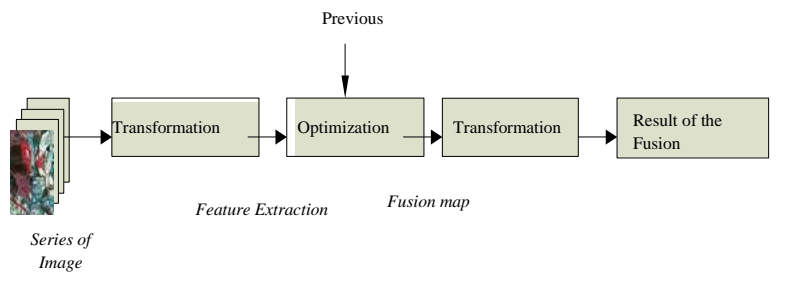

Figure 5: Block diagram of a fusion process

\section{LIMITATIONS OF FUSION METHODS}

Before implementing and using a fusion algorithm it is important to take into account the purpose of the application and the type of data based on it define the fusion technique more adequate and fusion rule to carry out the combination of data. For example, in medical images, the first thing to be defined is the medical modalities with which we want to work. The next stage is the choice of an appropriate level of fusion since the Pre-preprocessing stages depend on this. Pixel-based fusion approaches deal with huge amount of data whereby there is a considerable amount of computation time. The fusion at the level of features use equivalent frameworks, which make geometry a matter less critical However, pixel-based approaches have the benefit over level characteristics or decision to use the data in its original form, which avoids losses of information that occur during the feature extraction process. It is easy to create universal assumptions about the superiority of a technical fusion. On the other hand, the features of the fused data are heavily dependent on the fusion method applied and preprocessing used. The restrictions are connected to the disruption of the spectral content of the input data and effects of blurring when images with a low signal to noise ratio are introduced. In the case of multimodal image fusion, the problem of reconstruction of local intensities of the fused image is present because the images of input are attained from different multimodal sensors, which have different ranges and values of intensities [20].

\section{PROPOSED METHOD}

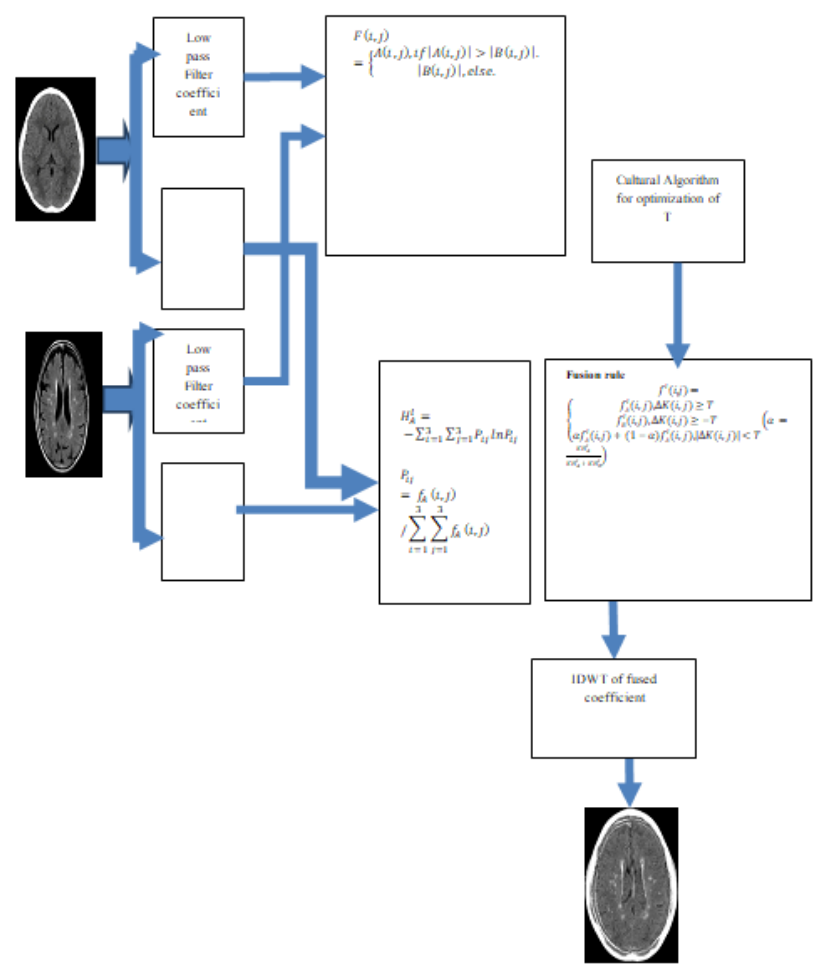

Figure 6: Proposed method of CT and MRI image fusion with DWT and cultural algorithm optimization

\section{A. Cultural Algorithm-DWT Based Fusion}

1. Decompose MRI image to 1 level using "sym4" 2 dimensional DWT.

2. Decompose CT image to 1 level using "sym4" 2 dimensional DWT.

3. For approximate (low frequency) coefficient, we use equation (5).

$$
F(i, j)=\left\{\begin{array}{c}
A(i, j), \text { if }|A(i, j)|>|B(i, j)| . \\
|B(i, j)|, \text { else. }
\end{array}\right.
$$

Where $F(i, j), A(i, j)$, and $B(i, j)$ are frequency coefficients of fused image $F$.

4. For each High-frequency sub-band of both image, do the following:

a. A selection of region with a window of $3 * 3$ and computed regional information entropy using equation (9) and (10).

$$
\begin{gathered}
H_{A}^{l}-\sum_{i=1}^{3} \sum_{j=1}^{3} P_{i j} \ln P_{i j} \\
P_{i j}=f_{A}(i, j) / \sum_{i=1}^{3} \sum_{j=1}^{3} f_{A}(i, j)
\end{gathered}
$$

Where $H_{A}^{l}$ is the entropy of the image. $P_{i j}$ is the gray value probability of a point $(i, j)$ in the selected image according to window image, $f_{A}(i, j)$ is the gray value of point $(i, j)$.

b. Computed regional information entropy contrast degree using equation (11). 


$$
K H_{A}^{l}(i, j)=\frac{H_{A}^{l}(i, j)}{H_{A}^{H}(i, j)+H_{A}^{V}(i, j)+H_{A}^{D}(i, j)}
$$

Where $\quad K H_{A}^{l}(i, j)$ represents contrast degree of image.

c. Compared contrast the degree of two images using equation (12).

$$
\Delta K(i, j)=K H_{A}^{l}(i, j)-K H_{B}^{l}(i, j)
$$

5. Now use (Cultural) to find threshold value $\mathrm{T}$ (to be used in equation (13) for high-frequency subband:

$$
f^{l}(i, j)=\left\{\begin{array}{c}
f_{A}^{l}(i, j), \Delta K(i, j) \geq T \\
f_{B}^{l}(i, j), \Delta K(i, j) \geq-T \\
\alpha f_{A}^{l}(i, j)+(1-\alpha) f_{A}^{l}(i, j),|\Delta K(i, j)|<T \\
\left.\frac{K H_{A}^{l}}{K H_{A}^{l}+K H_{B}^{l}}\right)
\end{array}(\alpha=\right.
$$

a. Fitness function

$\max E\left(F_{T}\right)=\operatorname{Entropy}\left(I D W T 2\left(F_{T}\right)\right)$

Where $F_{T}$ is the fused image by equation (14) using the optimized value of $\mathrm{T}$. b. The value of $\mathrm{T}$ which will give maximum entropy in the fused image will be the final value $(0<\mathrm{T}<1)$;

6. After finding $\mathrm{T}$, Fuse the coefficients using equation (14).

7. Do IDWT using "sym4" 2 dimensional DWT.

8. Quality Assessment

a. Fusion Quality Parameter

i. Entropy

ii. Find the above parameters for MRI, CT, and Fused image to show fusion Quality since we don't have any reference.

9. Output

\begin{tabular}{|c|c|c|c|}
\hline & Parameter & Formula & Explanation \\
\hline 1. & $\begin{array}{l}\text { Mutual information } \\
\text { based on Quality } \\
\text { Metrics }\end{array}$ & $\begin{array}{l}M I_{F A}(f, a) \\
=\sum_{f, a} P_{F A}(f, a) \log \left[\frac{P_{F A}(f, a)}{P_{F}(f) P_{A}(a)}\right]\end{array}$ & $\begin{array}{l}\text { Mutual information (MI) is a notion of the theory of information which } \\
\text { proposed as an evaluation measure of the fusion of images in the absence } \\
\text { of a reference image (ground truth). }\end{array}$ \\
\hline 2 & $\begin{array}{l}\text { Standard deviation } \\
\text { (STD) }\end{array}$ & $S T D=\sqrt{\frac{\sum_{i=0}^{N-1} \sum_{j=0}^{M-1} F(i, j)}{N M}}$ & $\begin{array}{l}\text { The STD is the square root of the variance (standard deviation), the } \\
\text { variance of an image reflects the scatter gradient between the grayscale } \\
\text { values and mean value of grayscale, the higher the STD, the more a } \\
\text { dispersion }\end{array}$ \\
\hline 3 & Entropy & $E N=-\sum_{i=0}^{L-1} P_{f}(i) \log _{2} P_{f}(i)$ & $\begin{array}{l}\text { Entropy is a criterion that measures the degree of information in the } \\
\text { image, the higher the EN, the more the image diffuses information }\end{array}$ \\
\hline 4 & $\begin{array}{l}\text { Structural Similarity } \\
\text { Index (SSIM) }\end{array}$ & $\begin{array}{l}\operatorname{SSIM}(p, q) \\
=\frac{\left(2 \mu_{p} \mu_{q}+c 1\right)\left(\sigma_{p q}+c 2\right)}{\left(\mu_{p}^{2}+\mu_{q}^{2}+c 1\right)\left(\sigma_{p}^{2}+\sigma_{q}^{2}+c 2\right)}\end{array}$ & $\begin{array}{l}\mu_{p} \text { the average of } p \\
\mu_{q} \text { the average of } q \\
\sigma_{p}^{2} \text { the variance of } p \\
\sigma_{q}^{2} \text { the variance of } q \\
\sigma_{p q} \text { the covariance of } p \text { and } q \\
c 1=\left(k_{1} L\right)^{2}, c 2=\left(k_{1} L\right)^{2} \text { two variables to stabilize the division with weak } \\
\text { denominator } \\
L \text { is a dynamic range of pixel values } \\
k_{1}=0.01 \text { and } k_{2}=0.03 \text { by default }\end{array}$ \\
\hline 5 & $\begin{array}{l}\text { Structural Content } \\
\text { (SC) }\end{array}$ & $S C=\frac{\sum_{i=1}^{m} \sum_{j=1}^{n}\left(A_{i j}\right)^{2}}{\sum_{i=1}^{m} \sum_{j=1}^{n}\left(B_{i j}\right)^{2}}$ & $\begin{array}{l}\text { It shows the connection between the net sum of the square of the normal } \\
\text { information and the aggregate sum of the square of the information } \\
\text { acquired }\end{array}$ \\
\hline 6 & $\begin{array}{l}\text { Average difference } \\
(A D):\end{array}$ & $A D=\frac{1}{m n} \sum_{i=1}^{m} \sum_{j=1}^{n}\left(\left|A_{i j}-B_{i j}\right|\right)$ & $\begin{array}{l}\text { The image intensity of both images at corresponding points are identical } \\
\text { when recorded from the same source. }\end{array}$ \\
\hline 7 & $\begin{array}{l}\text { Maximum difference } \\
\text { (MD): }\end{array}$ & $M D=\max \left(\left|A_{i j}-B_{i j}\right|\right)$, & $\begin{array}{l}\text { The distinction between every one of the densities of relating pixels is } \\
\text { determined. }\end{array}$ \\
\hline 8 & $\begin{array}{l}\text { Normalized Absolute } \\
\text { Error (NAE): }\end{array}$ & $N A E=\frac{\sum_{i=1}^{m} \sum_{j=1}^{n}\left(\left|A_{i j}-B_{i j}\right|\right)}{\sum_{i=1}^{m} \sum_{j=1}^{n}\left(A_{i j}\right)}$ & $\begin{array}{l}\text { It is the net aggregate association between the perfect values and the error } \\
\text { values. }\end{array}$ \\
\hline
\end{tabular}

a. Fused image

\section{B. Performance Evaluation Parameters}

Table 1: Evaluation parameters

\section{VII.RESULTS AND DISCUSSION}

Subject Images: eight subject MRI and CT images are taken from hospital with following details of modality and dimension.

Table 2: Input description

\begin{tabular}{|c|c|c|c|c|}
\hline Subject & Modality & Dimensions & bytes & Class \\
\hline \multirow{2}{*}{1} & MRI & $503 \times 813 \times 3$ & 1226817 & Uint8 \\
\cline { 2 - 5 } & CT & $442 \times 442 \times 3$ & 586072 & Uint8 \\
\hline \multirow{2}{*}{2} & MRI & $532 \times 605 \times 3$ & 965580 & Uint8 \\
\cline { 2 - 5 } & CT & $497 \times 532 \times 3$ & 793212 & Uint8 \\
\hline \multirow{2}{*}{3} & MRI & $631 \times 532 \times 3$ & 1007076 & Uint8 \\
\cline { 2 - 5 } & CT & $584 \times 524 \times 3$ & 918048 & Uint8 \\
\hline
\end{tabular}

\begin{tabular}{|c|c|c|c|c|}
\hline \multirow{2}{*}{4} & MRI & $408 \times 349 \times 3$ & 413292 & Uint8 \\
\cline { 2 - 5 } & CT & $404 \times 320 \times 3$ & 387840 & Uint8 \\
\hline \multirow{2}{*}{5} & MRI & $465 \times 802 \times 3$ & 987714 & Uint8 \\
\cline { 2 - 5 } & CT & $546 \times 603 \times 3$ & 874776 & Uint8 \\
\hline \multirow{2}{*}{6} & MRI & $508 \times 374 \times 3$ & 566703 & Uint8 \\
\cline { 2 - 5 } & CT & $453 \times 417 \times 3$ & 887714 & Uint8 \\
\hline \multirow{2}{*}{7} & MRI & $407 \times 872 \times 3$ & 625152 & Uint8 \\
\cline { 2 - 5 } & CT & $568 \times 550 \times 3$ & 937200 & Uint8 \\
\hline \multirow{2}{*}{8} & MRI & $221 \times 228 \times 3$ & 151164 & Uint8 \\
\cline { 2 - 5 } & CT & $285 \times 288 \times 3$ & 21880 & Uint8 \\
\hline
\end{tabular}



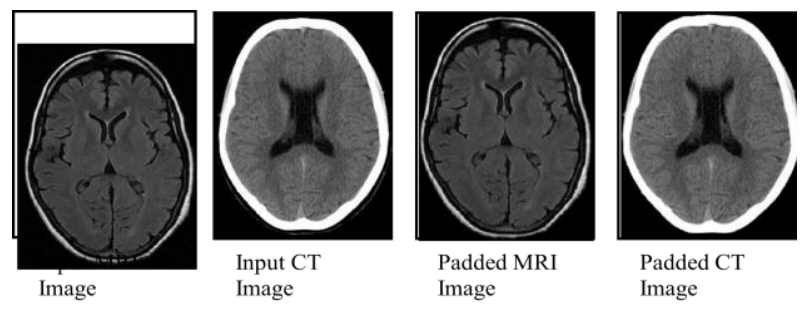

Figure 7: Subject-11

Table 3: Subject 1 Results 1 - Fusion Algorithms

\begin{tabular}{|c|c|c|c|c|c|}
\hline & AD & MD & NAE & NK & SC \\
\hline $\begin{array}{c}\text { P. } \\
\text { Simpl } \\
\text { e }\end{array}$ & 1 & $\begin{array}{c}6007734 \\
3\end{array}$ & 683 & $\begin{array}{c}0.86438993 \\
3\end{array}$ & $\begin{array}{c}1.19381413 \\
3\end{array}$ \\
\hline VMax & $\begin{array}{c}1.37715466 \\
6 \\
\end{array}$ & 0 & $\begin{array}{c}60412614 \\
3 \\
\end{array}$ & $\begin{array}{c}1.16620681 \\
7 \\
\end{array}$ & $\begin{array}{c}0.62552266 \\
9 \\
\end{array}$ \\
\hline VMin & $\begin{array}{c}1.37715466 \\
6 \\
\end{array}$ & $\begin{array}{c}1.72015468 \\
6 \\
\end{array}$ & $\begin{array}{c}0.55596753 \\
2 \\
\end{array}$ & $\begin{array}{c}0.56257304 \\
9 \\
\end{array}$ & $\begin{array}{c}2.49167211 \\
8 \\
\end{array}$ \\
\hline DWT & $\begin{array}{c}0,13388635 \\
7 \\
\end{array}$ & $\begin{array}{c}0.76709750 \\
3 \\
\end{array}$ & $\begin{array}{c}0.54810601 \\
1 \\
\end{array}$ & $\begin{array}{c}0.89983700 \\
9 \\
\end{array}$ & $\begin{array}{c}1.04617204 \\
7 \\
\end{array}$ \\
\hline PCA & $\begin{array}{c}2.43188 \mathrm{E}- \\
06\end{array}$ & $\begin{array}{c}0.86765954 \\
5 \\
\end{array}$ & $\begin{array}{c}0.58248073 \\
8 \\
\end{array}$ & $\begin{array}{c}0.87443427 \\
5 \\
\end{array}$ & $\begin{array}{c}1.14438183 \\
1 \\
\end{array}$ \\
\hline
\end{tabular}

Table 4: Subject 2 Results 1 - Fusion Algorithms

\begin{tabular}{|c|c|c|c|c|c|}
\hline \multicolumn{6}{|c|}{ subject 2 - MRI-CT } \\
\hline & AD & MD & NAE & NK & SC \\
\hline $\begin{array}{c}\text { P. } \\
\text { Simpl } \\
\text { e }\end{array}$ & $\begin{array}{c}2,22045 \mathrm{E}- \\
17\end{array}$ & $\begin{array}{c}0,89423472 \\
8\end{array}$ & 0,49486791 & $\begin{array}{c}0,88360044 \\
4\end{array}$ & $\begin{array}{c}1,16045401 \\
8\end{array}$ \\
\hline VMax & $\begin{array}{c}1,21335817 \\
8 \\
\end{array}$ & 0 & $\begin{array}{c}0,50253047 \\
9 \\
\end{array}$ & $\begin{array}{c}1,16938736 \\
1 \\
\end{array}$ & $\begin{array}{c}0,63645116 \\
3 \\
\end{array}$ \\
\hline VMin & $\begin{array}{c}1,21335817 \\
8 \\
\end{array}$ & $\begin{array}{c}1,78846945 \\
6 \\
\end{array}$ & $\begin{array}{c}0,48720534 \\
1 \\
\end{array}$ & $\begin{array}{c}0,59781352 \\
8 \\
\end{array}$ & $\begin{array}{c}2,33215734 \\
7 \\
\end{array}$ \\
\hline DWT & 0,05892981 & $\begin{array}{c}0,83110436 \\
3 \\
\end{array}$ & $\begin{array}{c}0,48306444 \\
6 \\
\end{array}$ & 0,90865243 & $\begin{array}{c}1,06679917 \\
7 \\
\end{array}$ \\
\hline PCA & 1,76E-06 & $\begin{array}{c}0,96150709 \\
5\end{array}$ & $\begin{array}{c}0,50146831 \\
9\end{array}$ & $\begin{array}{c}0,89400124 \\
1\end{array}$ & $\begin{array}{c}1,11193583 \\
5\end{array}$ \\
\hline
\end{tabular}

Table 5: Subject 3 Results 1 - Fusion Algorithms

\begin{tabular}{|c|c|c|c|c|c|}
\hline \multicolumn{6}{|c|}{ subject 3- MRI-CT } \\
\hline & AD & MD & NAE & NK & SC \\
\hline $\begin{array}{c}\text { P. } \\
\text { Siml } \\
\text { e }\end{array}$ & 0 & 0,89960970 & 0,50897975 & 0,87824379 & 1,13999648 \\
& & 3 & 2 & 3 & 9 \\
VMax & $\begin{array}{c}1,50303949 \\
9\end{array}$ & 0 & 0,55733277 & 1,14242984 & 0,6543722 \\
VMin & 1,50303949 & 1,79921940 & 0,46062672 & 0,61405773 & 2,11946173 \\
\hline DWT & 0,12992530 & 0,85897663 & 0,48761898 & 0,88213466 & 1,12086021 \\
& 4 & 3 & 7 & 8 & 7 \\
\hline & $2,55184 \mathrm{E}-$ & 0,91457052 & 0,51030698 & 0,89012028 & 1,09439210 \\
PCA & 06 & 9 & 1 & 2 & 7 \\
\hline
\end{tabular}

Table 6: Subject 4 Results 1 - Fusion Algorithms

\begin{tabular}{|c|c|c|c|c|c|}
\hline \multicolumn{6}{|c|}{ subject 4-MRI-CT } \\
\hline & AD & MD & NAE & NK & SC \\
\hline $\begin{array}{c}\text { P. } \\
\text { Simpl } \\
\text { e }\end{array}$ & 0 & 0,91854807 & 0,50986214 & 0,88861203 & 1,12862461 \\
6 & 9 & 1 \\
\hline
\end{tabular}

\begin{tabular}{|c|c|c|c|c|c|}
\hline & 1,63048070 & & 0,58758372 & 1,14609076 & 0,6602548 \\
VMax & 3 & 0 & 1 & 3 & 7 \\
\hline VMin & 1,63048070 & 1,83709615 & 0,43214057 & 0,63113331 & 2,06001499 \\
& 3 & 9 & 1 & 6 & 3 \\
\hline & $\begin{array}{c}0,09862585 \\
\text { DWT }\end{array}$ & $\begin{array}{c}0,89633523 \\
0\end{array}$ & $\begin{array}{c}0,49527151 \\
0,88523924\end{array}$ & $\begin{array}{c}1,13811056 \\
5\end{array}$ \\
\hline & $\begin{array}{c}3,93668 \mathrm{E}- \\
\text { PCA }\end{array}$ & 0,98714891 & 5 & 3 & 5 \\
\hline \multicolumn{5}{|r|}{} \\
\hline
\end{tabular}

Table 7: Subject 5 Results 1 - Fusion Algorithms subject 5- MRI-CT

\begin{tabular}{|c|c|c|c|c|c|}
\hline & AD & MD & NAE & NK & SC \\
\hline $\begin{array}{c}\text { P. } \\
\text { Simpl } \\
\text { e }\end{array}$ & $\begin{array}{c}1,77636 \mathrm{E}- \\
16\end{array}$ & $\begin{array}{c}0,87162033 \\
1\end{array}$ & $\begin{array}{c}0,52192617 \\
8\end{array}$ & $\begin{array}{c}0,90106200 \\
1\end{array}$ & $\begin{array}{c}1,11817099 \\
3\end{array}$ \\
\hline VMax & $\begin{array}{c}1,76541544 \\
2\end{array}$ & 0 & $\begin{array}{c}0,64058299 \\
8\end{array}$ & $\begin{array}{c}1,1284131 \\
5\end{array}$ & $\begin{array}{c}0,68605626 \\
7\end{array}$ \\
\hline VMin & $\begin{array}{c}1,76541544 \\
2\end{array}$ & $\begin{array}{c}1,74324066 \\
2\end{array}$ & $\begin{array}{c}0,40326935 \\
7\end{array}$ & $\begin{array}{c}0,67371085 \\
3\end{array}$ & $\begin{array}{c}1,84367954 \\
1\end{array}$ \\
\hline DWT & $\begin{array}{c}0,16639439 \\
6 \\
\end{array}$ & $\begin{array}{c}0,84965410 \\
6 \\
\end{array}$ & $\begin{array}{c}0,49897457 \\
3 \\
\end{array}$ & $\begin{array}{c}0,89403963 \\
5 \\
\end{array}$ & $\begin{array}{c}1,13799967 \\
1 \\
\end{array}$ \\
\hline PCA & $1,08 \mathrm{E}-06$ & 0,88178473 & $\begin{array}{c}0,52116696 \\
8\end{array}$ & $\begin{array}{c}0,91402067 \\
9\end{array}$ & $\begin{array}{c}1,07730977 \\
9\end{array}$ \\
\hline
\end{tabular}

Table 8: Subject 6 Results 1 - Fusion Algorithms

\begin{tabular}{|c|c|c|c|c|c|}
\hline \multicolumn{7}{|c|}{ subject 6 - MRI-CT } \\
\hline & AD & MD & NAE & NK & SC \\
\hline P. & & & & & \\
Simpl & $8,88178 \mathrm{E}-$ & 0,88355662 & 0,54162531 & 0,90122345 & 1,12336316 \\
e & 17 & 7 & 2 & 3 & 6 \\
\hline VMax & 1,78848834 & 0 & 0,67383464 & 1,13712903 & 0,67790724 \\
& 4 & & 6 & 8 & 8 \\
\hline & 1,78848834 & 1,76711325 & 0,40941597 & 0,66531786 & 1,90522661 \\
VMin & 4 & 4 & 8 & 7 & 8 \\
\hline & 0,14459482 & 0,86621836 & 0,52067754 & 0,89369099 & 1,14571544 \\
DWT & 7 & 3 & 1 & 6 & 1 \\
\hline & & 0,81956149 & 0,53969416 & 0,91430749 & 1,08305136 \\
PCA & $8,2025 \mathrm{E}-06$ & 3 & 9 & 1 & 7 \\
\hline
\end{tabular}

Table 9: Subject 7 Results 1 - Fusion Algorithms

\begin{tabular}{|c|c|c|c|c|c|}
\hline \multicolumn{6}{|c|}{ subject 7 -MRI-CT } \\
\hline & AD & MD & NAE & NK & SC \\
\hline $\begin{array}{c}\text { P. } \\
\text { Simpl } \\
\text { e }\end{array}$ & 0 & $\begin{array}{c}0,87876289 \\
9\end{array}$ & $\begin{array}{c}0,43373062 \\
1\end{array}$ & $\begin{array}{c}0,91552628 \\
5\end{array}$ & $\begin{array}{c}1,09532076 \\
9\end{array}$ \\
\hline VMax & $\begin{array}{c}1,3654917 \\
7 \\
\end{array}$ & 0 & $\begin{array}{c}0,48342961 \\
6 \\
\end{array}$ & $\begin{array}{c}1,14288015 \\
8 \\
\end{array}$ & $\begin{array}{c}0,68814466 \\
1 \\
\end{array}$ \\
\hline VMin & $\begin{array}{c}1,3654917 \\
7 \\
\end{array}$ & $\begin{array}{c}1,75752579 \\
8 \\
\end{array}$ & $\begin{array}{c}0,38403162 \\
6 \\
\end{array}$ & $\begin{array}{c}0,68817241 \\
1 \\
\end{array}$ & $\begin{array}{c}1,82876478 \\
5 \\
\end{array}$ \\
\hline DWT & $\begin{array}{c}0,03935251 \\
7 \\
\end{array}$ & $\begin{array}{c}0,8696700 \\
3\end{array}$ & $\begin{array}{c}0,42856575 \\
8 \\
\end{array}$ & $\begin{array}{c}0,91374426 \\
2 \\
\end{array}$ & $\begin{array}{c}1,10017462 \\
9 \\
\end{array}$ \\
\hline PCA & $3,84 \mathrm{E}-06$ & $\begin{array}{c}0,86930590 \\
8 \\
\end{array}$ & $\begin{array}{c}0,43821745 \\
5\end{array}$ & 0,92795222 & $\begin{array}{c}1,05553640 \\
3 \\
\end{array}$ \\
\hline
\end{tabular}


Table 10: Subject 8 Results 1 - Fusion Algorithms

\begin{tabular}{|c|c|c|c|c|c|}
\hline \multicolumn{6}{|c|}{ subject 8 - MRI-CT } \\
\hline & AD & MD & NAE & NK & SC \\
\hline $\begin{array}{c}\text { P. } \\
\text { Simpl } \\
\text { e }\end{array}$ & $\begin{array}{c}8,88178 \mathrm{E}- \\
17\end{array}$ & $\begin{array}{c}0,84952752 \\
2\end{array}$ & $\begin{array}{c}0,48069325 \\
9\end{array}$ & $\begin{array}{c}0,91619330 \\
2\end{array}$ & $\begin{array}{c}1,10485614 \\
7\end{array}$ \\
\hline VMax & $\begin{array}{c}1,61069171 \\
2\end{array}$ & 0 & $\begin{array}{c}0,56824161 \\
8\end{array}$ & $\begin{array}{c}1,14944889 \\
9\end{array}$ & $\begin{array}{c}0,68225774 \\
2\end{array}$ \\
\hline VMin & $\begin{array}{c}1,61069171 \\
2\end{array}$ & $\begin{array}{c}1,69905504 \\
4\end{array}$ & $\begin{array}{c}0,39314490 \\
1\end{array}$ & $\begin{array}{c}0,68293770 \\
5\end{array}$ & $\begin{array}{c}1,87168384 \\
5\end{array}$ \\
\hline DWT & $\begin{array}{c}0,03301167 \\
7\end{array}$ & $\begin{array}{c}0,84427295 \\
5\end{array}$ & $\begin{array}{c}0,47633871 \\
6\end{array}$ & $\begin{array}{c}0,91402336 \\
7\end{array}$ & $\begin{array}{c}1,11113804 \\
5\end{array}$ \\
\hline PCA & $\begin{array}{c}7,16051 \mathrm{E}- \\
06\end{array}$ & $\begin{array}{c}0,85915039 \\
5\end{array}$ & $\begin{array}{c}0,48049307 \\
4\end{array}$ & $\begin{array}{c}0,92751719 \\
5\end{array}$ & $\begin{array}{c}1,07071544 \\
7\end{array}$ \\
\hline
\end{tabular}

Given the results, some initial conclusions can be drawn about the work:

When there is about merging images using wavelet algorithms in the context of the medical image, there is no need to go deeper than the first level in the wavelet decomposition, since the results get significantly worse as is going to the next level. In this study, the third level of decomposition has been reached, although only in the first one, useful results have been obtained for a diagnostic examination.

Calculating the arithmetic mean on wavelet coefficients does not provide any advantage over said calculated mean in the spatial domain directly over gray intensity levels. In spite of being a simple calculation and that could seem a fundamental algorithm, both numerical and visual results have not been bad at all.

It has verified that how the values of the variances and the differences between them vary greatly depending on where the window that the matrix is traveling is located. Since for example the window of the images in an area where all the pixels have the same value (and therefore the variance is zero), while in the other image there are abrupt changes in intensity (which gives a high variance), the difference between variances offers a high score that is well above the threshold. Then the differences between variances fluctuate very sharply and in general, this method of fusion is not sensitive to the established threshold, whatever its value.

The variation coefficient, on the other hand, offers more original values between both images, and above all more homogeneous during the window's travel, and is much more sensitive to changes in the threshold. The best numerical values are obtained for high threshold values, although it should be noted that increasing the threshold level is causing a greater number of calculations of the arithmetic mean between the central coefficients of the threshold.

There are no significant variations in the data obtained when comparing the activity level calculations on the whole image or a coefficient window.

The chosen simple fusion algorithm is the Principal Component Analysis (PCA) and for the pyramidal decomposition algorithms the Discrete Wavelet Transform DWT.

Therefore, results obtained from a quantitative analysis performed on two categories of images (CT and MRI). As previously mentioned, the images used are medical images of an X-ray tomography (CT) and an MRI of the same organ, section, and cut.

In the case of fusion using both wavelets and pyramidal decomposition techniques, a comparison is first made between each of the fusion algorithms up to the $3^{\text {rd }}$ level, to determine the best level of decomposition, making a comparison with the ideal measurements and the obtained measurements of the image quality for each method of pyramidal and wavelet fusion.

The images that must merge and processed before the application of the fusion algorithm. Pre-processing comprises of the image resizing and image registration. The images generated by diverse modalities can have diverse orientations and, therefore, it is necessary to register them before they are merged. In image resizing, the sizes of the images may vary, so before the merging, it is essential to change the size of the scans so that both the scans have the same size.

This resizing is achieved by converting the less sized image with the duplicate rows and columns matrix. Image enhancement, if both or any of the images are not grayscale, then you want them to be converted to grayscale. The next step that follows is the equalization of the image histogram so that the contrast of the image is improved and both images have a similar range of values for their coefficients.

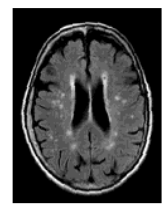

Figure 8: Registered

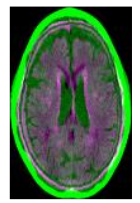

Figure 9: Fused
Table 11: Subject1-MRI-CT-image fusion result

\begin{tabular}{|c|c|c|c|}
\hline & PCA & DWT & Cultural Optimized DWT \\
\hline MI & 2.7521 & 3.9745 & 4.145 \\
\hline Entropy & 4.9423 & 5.1536 & 5.9634 \\
\hline STD & 45.0794 & 47.7946 & 48.345 \\
\hline SSIM & 0.8136 & 0.8329 & 0.8573 \\
\hline
\end{tabular}

\section{A. Subjective Assessment}

Subjective assessment by radiologists is another system and utilized in estimating the quality of the fused image. The abstract test enthusiastically watches steadfastness and in the meantime, studies image clarity. When playing out the subjective analysis, the radiologist centers on the similitude among the fused image and the first scan. When watching such facts, data loss can't be acknowledged. The subjective analysis led by a radiologist (R) from Mahatma Gandhi Hospital (Imaging Center) and 2 specialists as Analyzer (Ob1, Ob2) from the medical clinic. The Graphical 8 subjects of the source scan and their relating fused images are noticeable in the outcomes one by one relying upon the source scan and the calculation utilized. In the assessment procedure, we arbitrarily chose the fused image amidst the five calculations. The state of the calculation isn't known for the radiologist/analyzer.

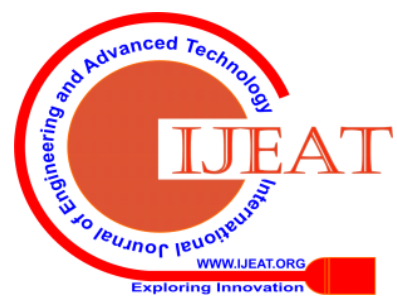


The radiologist/analyzer can rate the calculation ranges 1 to 10 dependent on the visual appearance. The observation by the radiologist/analyzer appears in Figure 10, Table 12. The terms utilized for valuation are Total Number of Evaluation (TNE) and Average of Evaluation (AvgE). TNE demonstrates the number of assessments led by the radiologist/analyzer for each image arbitrarily, and AvgE demonstrates the normal of assessments gotten by the TNE.

Table 12: Subjective evaluation of subject-1 fused images by the analyzer

\begin{tabular}{|c|c|c|c|c|c|c|}
\hline $\begin{array}{c}\text { Name of } \\
\text { Analyzer }\end{array}$ & Evaluation & A1 & A2 & A3 & A4 & A5 \\
\hline \multirow{2}{*}{ Ob1 } & TNE & 3 & 2 & 3 & 4 & 5 \\
\cline { 2 - 7 } & AvgE & 6.5 & 7 & 7 & 8.5 & 9 \\
\hline \multirow{2}{*}{ Ob2 } & TNE & 3 & 3 & 3 & 3 & 5 \\
\cline { 2 - 7 } & AvgE & 7 & 6 & 7 & 7.5 & 8 \\
\hline
\end{tabular}

\section{Radiologist Evaluation}

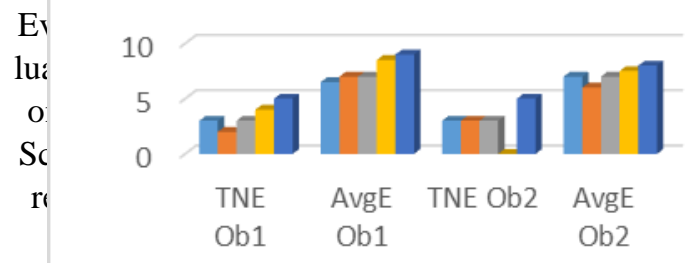

$\because \mathrm{A} 1 \square \mathrm{A} 2 \square \mathrm{A} 3$

Figure 10: Subjective assessment by the radiologist for Subject 1

\section{CONCLUSION}

This paper presents a description of the main models to perform MRI \& CT image fusion, by implementing algorithms such as; Simple Average, Maximum Value, Value Minimum, Principal Component Analysis, Discrete DWT Wavelet Transform, in the MATLAB environment, which is easy through image quality measures such as; Average difference (AD), Maximum difference (MD), Absolute error Normalized (NAE), Normalized Cross Correlation (NK), and Structural Content (SC), obtaining results close to the ideal values for each of the tests, between 0 and 1 contrasting the quality of the merged image.

Further cultural optimized entropy based algorithm has been develop to fuse CT and MRI images, on different patient data. The proposed method claims better outcome as compared to conventional method under different performance evaluation parameters. The archived results with respect to MI, SSIM, Entropy and STD form proposed methods outperform then PCA, DWT. The claimed SSIM is 0.8573 for cultural optimized DWT method. The simulation results are better with high quality fused images when evaluated with objective and subjective metrics.

The functionality of the module for internal analysis was evaluated, and ground truth is referred by observer, TNE and AVE is claimed as performance parameter, the calculated results claims the robustness of proposed method, which is superior then other conventional methods.

\section{REFERENCES}

1. Aktar, M.N., Lambert, A.J. and Pickering, M., 2018. An automatic fusion algorithm for multi-modal medical images. Computer Methods in Biomechanics and Biomedical Engineering: Imaging \& Visualization, 6(5), pp.584-598.

2. Nair, R.R. and Singh, T., 2019. Multi-sensor medical image fusion using pyramid-based DWT: a multi-resolution approach. IET Image Processing.

3. Du, J., Li, W., Lu, K. and Xiao, B., 2016. An overview of multimodal medical image fusion. Neurocomputing, 215, pp.3-20.

4. Rajalingam, B., Priya, R. and Bhavani, R., 2019. Hybrid Multimodal Medical Image Fusion Using Combination of Transform Techniques for Disease Analysis. Procedia Computer Science, 152, pp.150-157.

5. Sandhya, S., Kumar, M.S. and Karthikeyan, L., 2019. A Hybrid Fusion of Multimodal Medical Images for the Enhancement of Visual Quality in Medical Diagnosis. In Computer Aided Intervention and Diagnostics in Clinical and Medical Images (pp. 61-70). Springer, Cham.

6. Du, J., Li, W. and Xiao, B., 2018. Fusion of anatomical and functional images using parallel saliency features. Information Sciences, 430, pp.567-576.

7. Bhatnagar, G., Wu, Q.J. and Liu, Z., 2015. A new contrast based multimodal medical image fusion framework. Neurocomputing, 157, pp.143-152.

8. Bhateja, V., Krishn, A. and Sahu, A., 2016. Medical image fusion in curvelet domain employing PCA and maximum selection rule. In Proceedings of the Second International Conference on Computer and Communication Technologies (pp. 1-9). Springer, New Delhi.

9. Du, J., Li, W., Xiao, B. and Nawaz, Q., 2016. Union Laplacian pyramid with multiple features for medical image fusion. Neurocomputing, 194, pp.326-339.

10. Moin, A., Bhateja, V. and Srivastava, A., 2016, March. Multispectral medical image fusion using PCA in wavelet domain. In Proceedings of the Second International Conference on Information and Communication Technology for Competitive Strategies (p. 87). ACM.

11. Zhu, Z., Yin, H., Chai, Y., Li, Y. and Qi, G., 2018. A novel multimodality image fusion method based on image decomposition and sparse representation. Information Sciences, 432, pp.516-529.

12. Rajinikanth, V., Satapathy, S.C., Dey, N. and Vijayarajan, R., 2018. DWT-PCA image fusion technique to improve segmentation accuracy in brain tumor analysis. In Microelectronics, Electromagnetics and Telecommunications (pp. 453-462). Springer, Singapore.

13. Zhang, N. and Wang, P., 2018, May. Multimodal Image Fusion for Brain Image Based on Nonsubsampled Shearlets Transform. In 2018 International Conference on Network, Communication, Computer Engineering (NCCE 2018). Atlantis Press.

14. Prasad, P., Subramani, S., Bhavana, V. and Krishnappa, H.K., 2019, March. Medical Image Fusion Techniques Using Discrete Wavelet Transform. In 2019 3rd International Conference on Computing Methodologies and Communication (ICCMC) (pp. 614-618). IEEE.

15. Du, J., Li, W. and Tan, H., 2019. Intrinsic Image DecompositionBased Grey and Pseudo-Color Medical Image Fusion. IEEE Access, 7, pp.56443-56456.

16. Krishn, A., Bhateja, V. and Sahu, A., 2015. PCA based medical image fusion in ridgelet domain. In Proceedings of the $3 r d$ International Conference on Frontiers of Intelligent Computing: Theory and Applications (FICTA) 2014 (pp. 475-482). Springer, Cham.

17. El-Hoseny, H.M., El Kareh, Z.Z., Mohamed, W.A., El Banby, G.M., Mahmoud, K.R., Faragallah, O.S., El-Rabaie, S., El-Madbouly, E. and El-Samie, F.E.A., 2019. An optimal wavelet-based multi-modality medical image fusion approach based on modified central force optimization and histogram matching. Multimedia Tools and Applications, pp.1-25.

18. Singh, S. and Anand, R.S., 2018. Ripplet domain fusion approach for CT and MR medical image information. Biomedical Signal Processing and Control, 46, pp.281-292.

19. Zhu, Y., Zhou, X., Li, X. and Zhang, R., 2019, April. Algorithm of Medical Image Fusion based on Laplasse Pyramid and PCA. In IOP Conference Series: Materials Science and Engineering (Vol. 490, No. 4, p. 042030). IOP Publishing.

20. Zhang, N., Wang, P. and Zong, X., 2018. A novel peripheral enhancement framework for CT and MRI image fusion in NSST domain. Journal of Medical Imaging and Health Informatics, 8(5), pp.891-899. 


\section{AUTHORS PROFILE}

Deepti Nathawat is a research scholar at the Department of Computer Science, Banasthali University. She completed her Masters in Software Engineering from Banasthali University. In research, her current interests include software testing, image processing, medical image processing, machine learning. She has completed her engineering in Computer Science from Rajasthan University and has 10 years of teaching experience. She is an Associate member of Institution of Engineer, India.

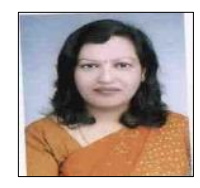

Dr. Manju Mandot is an Associate Professor and Director of Directorate of Jan Shikshan and Extension, Rajasthan Vidyapeeth (D) University. She completed her Ph.D (Computer Science) and has 27 years of teaching experience. Her research interest includes image processing, E- governance, women empowerment with technology. She is esteemed member of Computer Society of India.

Dr. Neelam Sharma is an Assistant Professor at the Department of Computer Science, Banasthali University. She has completed her PhD in Computer Science and has 13 years of teaching experience and her research interest includes machine learning, pattern recognition. 